\title{
〈 Case Report >
}

\section{Stress Fracture of the Fifth Metatarsal in Foot Deformity Secondary to Neuromuscular Disease}

\section{Experiences of Deformity Correction Treatment-A Report of 3 Cases and Review of the} Literature

\begin{abstract}
Fractures at the proximal metaphyso-diaphyseal junction of the fifth metatarsal are associated with high rates of delayed union. When these fractures are the result of repeated stress in patients with equinovarus hindfoot, which in turn is caused by neurological disorders, delayed union is the rule. Therefore, in neurological patients with stress fractures, optimal treatment would be to achieve a plantigrade foot enabling them to relieve the fifth metatarsal overload, which prevents the consolidation. We report 3 cases of fifth metatarsal stress fracture resulting from an equinovarus bindfoot deformity caused by a neuromuscular disease. Our surgical indication was
\end{abstract}

to correct the foot deformity with no direct action on the fracture. Once a good alignment (plantigrade foot) was obtained, stress causing the fracture disappeared, and union was achieved with optimal biomechanical function in all 3 fractures. When stress fracture of the fifth metatarsal is caused by a secondary foot deformity, treating the deformity can lead to bealing the fracture efficiently and should be considered prior to indicating surgical stabilization of the fracture itself. Primary treatment of
Jesús Payo-0llero, MD,

Fernando Álvarez Goenaga, MD,

Gotzon Elorriaga, Sagarduy, MD,

Alberto Ruiz Nasarre, MD,

Matías Alfonso Olmos-García, MD, PhD, and Carlos Villas Tomé, MD, PhD
Optimal treatment should address correction of the deformity leading to the stress fracture rather than the fracture itself."

DOI: 10.1177/1938640017744642. From University Clinic of Navarra, Orthopedic Surgery and Traumatology Department, Pamplona, Navarra, Spain (JP-0, MAO-G, CVT); San Rafael Hospital, Orthopedic Surgery and Traumatology Department, Barcelona, Catalunya, Spain (FAG, ARN); and University Cruces Hospital, Orthopedic Surgery and Traumatology Department, Baracaldo, Vizcaya, Spain (GES). Address correspondence to: Fernando Álvarez Goenaga, MD, San Rafael Hospital, Orthopedic Surgery and Traumatology Department, Passeig de la Vall d'Hebron, 107, Barcelona, Catalunya 08035, Spain; e-mail: alvarez173@gmail.com. 


\section{Introduction}

Fifth metatarsal fractures are the most common metatarsal fractures. ${ }^{1,2}$ Three main types of fractures may affect the proximal zone of the fifth metatarsal bone: (1) tuberosity avulsion fracture, (2) Jones's fracture, and (3) stress fracture. Jones's fracture was described in $1902^{3}$ as a transverse fracture at the junction of the diaphysis and the proximal metaphysis, with no extension distally beyond the fourth to fifth intermetatarsal joints. A stress fracture is defined as bone fatigue secondary to a repetitive load of the proximal fifth metatarsal. Both types of fractures, because of the anatomical proximity, have high rates of delayed union, nonunion, ${ }^{4}$ or refractures possibly favored by low blood supply in the proximal region. ${ }^{5-7}$

Chuckpaiwong et $\mathrm{al}^{8}$ did not consider it of interest to differentiate Jones's fracture from the proximal metaphyso-diaphyseal stress fracture because differences, sometimes subtle, may not be important from a prognostic point of view. Surgical treatment usually consists of anterograde intramedullary screw fixation. ${ }^{9-12}$ This approach reduces the rate of nonunion and shortens union time. ${ }^{13}$ In the literature, there are currently no references to foot morphology, and the type of fracture that is presented in this article is very rare. Refracture has been brought on by an inadequate synthesis material selection, ${ }^{14}$ high body mass index, plantar protrusion of the metatarsal head, varus hindfoot deformity, ${ }^{15}$ or premature return to sports activities. ${ }^{16}$

The aim of this study is to focus on the therapeutic approach to foot morphology in patients with a fifth metatarsal stress fracture and an equinovarus hindfoot deformity secondary to neurological disorders. In these cases, the treatment of the fracture with no correction of the deformity leads to therapeutic failure, and delayed union or nonunion of the fracture are the rule. Optimal treatment should address correction of the deformity leading to the stress fracture rather than the fracture itself. Moreover, correcting the deformity would improve

\section{Figure 1.}

Case 1: X-rays showing a stress fracture $(A)$ that was consolidated secondary to the intervention 40 days postoperatively (B). After a 4-year follow-up, the stress fracture remains consolidated (C).

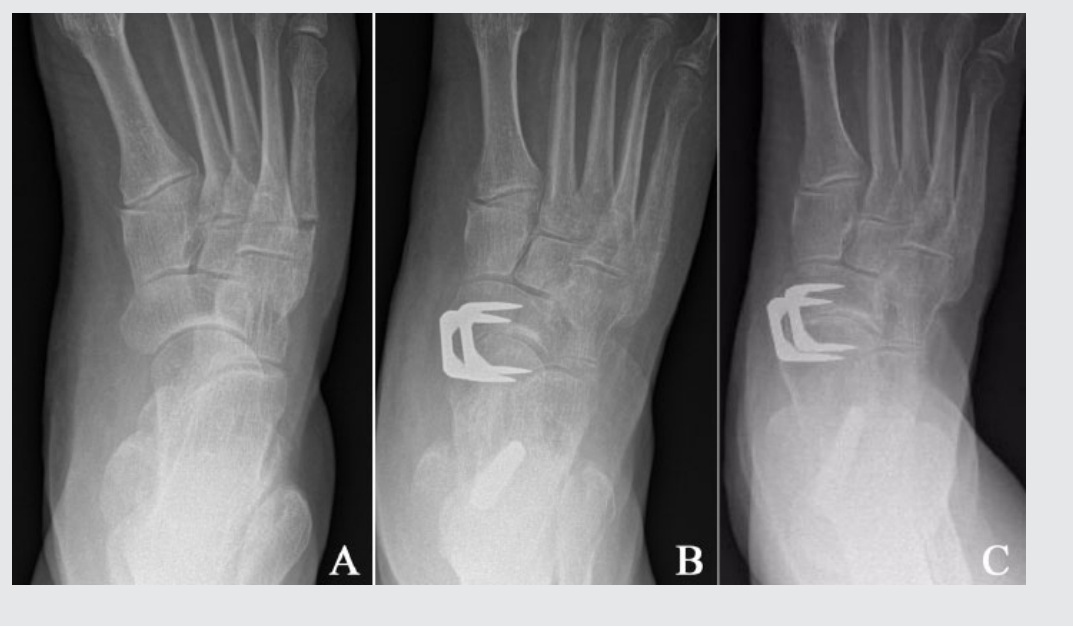

the biomechanics of the foot and the patient's gait.

\section{Case Reports \\ Case 1}

A 47-year-old woman was diagnosed with a proximal metaphyseal-diaphyseal junction fracture of the fifth metatarsal in 2013. At consultation, she had an equine and varus spastic right foot, secondary to a previous stroke (2 years previously), resulting in dystonic spastic hemiparesis. The patient complained of pain along the lateral edge of the foot, at the proximal zone of the fifth metatarsal. A diagnosis of fifth metatarsal stress fracture was made. Conservative treatment with a plaster cast for 3 months did not procure consolidation, and she continued to experience pain. Physical examination showed an equine and varus hindfoot deformity, with hyperkeratosis along the fifth metatarsal lateral border and under the metatarsal head (second rocker zone). Furthermore, there was hypertonia of the sural triceps underlying the equine deformity hypertonia of the tibialis posterior muscle with a relative muscular deficit of the tibialis anterior and peroneus muscles.
X-ray studies revealed an overall alteration of the anatomical structure of the foot (corresponding well with the equine and varus deformity) and a nonconsolidated transverse fracture at the metaphyseal-diaphyseal junction of the fifth metatarsal (Figure 1A).

The diagnosis was pseudoarthrosis of a stress fracture secondary to a dystonic spastic equine and varus hindfoot deformity. The patient was referred to our center for surgical treatment of the fracture, but we indicated double arthrodesis (talonavicular and subtalar joints) with a tibialis posterior transfer to the dorsal aspect of the foot and an Achilles tendon lengthening. After the operation, she continued to unload the extremity for 6 weeks with a subsequent plaster cast.

The plaster cast was removed once a bony union of the fracture was observed (Figure 1B). Weight-bearing was then allowed with a Cam Walker orthosis for a further 6 weeks. Three months following the operation, the evaluation of the double arthrodesis was favorable, and normal gait was allowed. Finally, a plantigrade foot without hyperpressure zones was obtained. After a 4-year follow-up (Figure 1C), the patient remains without pain at the fifth 
metatarsal region and only reports sporadic discomfort in the foot when hypertonia crises push her toes against the shoe. At the present moment, the foot is still plantigrade with no hyperpressure zones.

\section{Case 2}

A 38-year-old man consulted for pain and functional difficulties in the left foot throughout a period of 7 months. The patient had undergone multiple foot operations owing to a deformity secondary to Charcot-Marie-Tooth disease.

Physical examination revealed a cavus-varus hindfoot deformity with selective pain on the lateral edge of the foot-at the fifth metatarsal zone-where hyperkeratosis was noted. X-ray studies revealed a cavus-varus foot and a proximal transverse fracture of the fifth metatarsal at the metaphyso-diaphysis proximal junction (Figure 2A). Diagnosis was fifth metatarsal stress fracture on the left foot secondary to cavus-varus deformity.

Conservative treatment was applied, with a plaster cast for 6 weeks and a Cam Walker orthosis for a further 6 weeks. The fracture did not heal. Surgical treatment was then indicated and consisted of correcting the mechanical defect produced by the deformity. Arthrodesis of the subtalar joints (with screws) and the calcaneus-cuboid joint (with clamps) was then carried out; posterior tibialis tendon transfer to the dorsolateral part of the third cuneiform was associated with fibular tendons tenodesis and elevation osteotomy on the first metatarsal bone. Healing of the stress fracture was procured 3 months later (Figure 2B), leading to the patient being pain free. Two years later, the patient had a plantigrade foot and remained asymptomatic (Figure 2C).

\section{Case 3}

A 33-year-old man consulted with a proximal pseudoarthrosis of the fifth metatarsal on the left foot after failed conservative treatment with a plaster cast. The electromyographic study

\section{Figure 2.}

Case 2. Lateral X-rays showing a stress fracture $(A)$ and union of the fracture with new foot alignment 3 months after surgery (B). After a 2-year follow-up, the stress fracture remains consolidated (C).

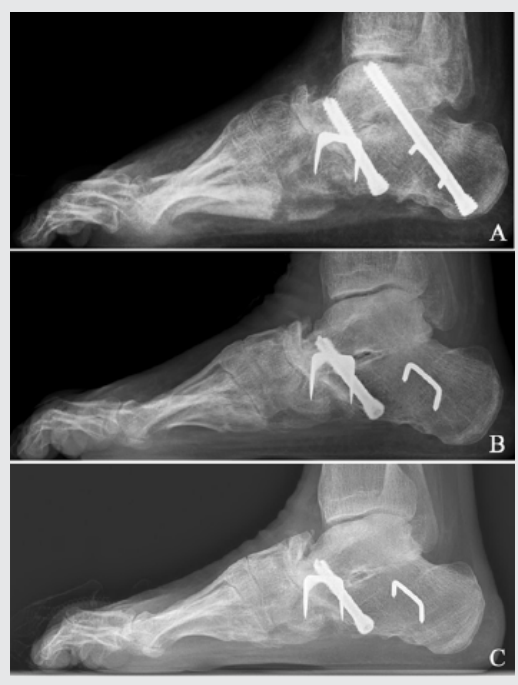

revealed a sensorimotor axonal polyneuropathy with motor involvement in both feet and gastrocnemius muscles. There was a previous family history of neurological diseases, but genetic studies did not show any abnormalities leading to a diagnosis of a specific condition.

Physical examination showed an equine deformity with a positive Coleman test in the left foot. Pes varus-cavus and equinovarus hindfoot generated high pressure around the fifth metatarsal and hyperkeratosis under its head in the left foot (Figure 3). In addition, there was hypertonia of the sural triceps, leading to an equine deformity. X-ray studies revealed an unconsolidated transverse fracture in the metaphyseal-diaphyseal junction at the base of the fifth metatarsal (Figure 4A).

Surgical treatment was carried out in 2 steps. The first consisted of a Dwyer osteotomy and calcaneal lateralization associated with elevation osteotomy on the first metatarsal bone, Achilles tendon lengthening, and plantar fasciotomy. In a postoperative control at 3 months, we observed consolidation of the

osteotomies and persistence of the fracture line in the fifth metatarsal. On physical examination, a plantigrade foot was observed with a varus axis correction. No further treatment was called for, and normal walking was allowed because the patient was asymptomatic. Then, 6 months after surgery, a new radiological control was performed, and complete healing of the fracture was observed (Figure 4B).

To improve dorsiflexion of the ankle, in a subsequent step, both a transfer of the posterior tibialis tendon to the dorsal aspect of the foot as well as a flexors tendon tenotomy were performed. Later, the same treatment was conducted on the right foot, which had never shown a stress fracture. After a 2-year follow-up (2 years for the left foot and 1 year for the right foot), the joint balance had improved in both feet (Figure 5), and the resolution of the stress fracture on the left foot persisted with the disappearance of the fracture line (Figure $4 \mathrm{C}$ ).

\section{Discussion}

The present study shows 3 cases of stress fracture secondary to equinovarus hindfoot deformities in neuromuscular disease, in which the fracture was successfully treated by correcting the deformity instead of acting directly on the fracture. The principle behind the therapeutic decision was to treat the deformity in order to correct the stress that caused the fracture. Although 3 cases of any specific topic may currently be considered to be a low-interest report for expert readers and, furthermore, the present topic seems to be traditionally (colloquially) well known by experienced surgeons, the fact remains that - in the past decades-the literature has offered a total lack of information, which may be useful for young foot surgeons when having to face similar problems and decide on the best therapeutic option.

Stress fracture of the fifth metatarsal may result from a plantar overload in the case of a progressive metatarsus adductus and equinovarus foot 


\section{Figure 3.}

Case 3: Posterior view of the ankle showing a cavus-varus hindfoot (A). The plantar views show high pressure around the fifth metatarsal and hyperkeratosis under its head (B).
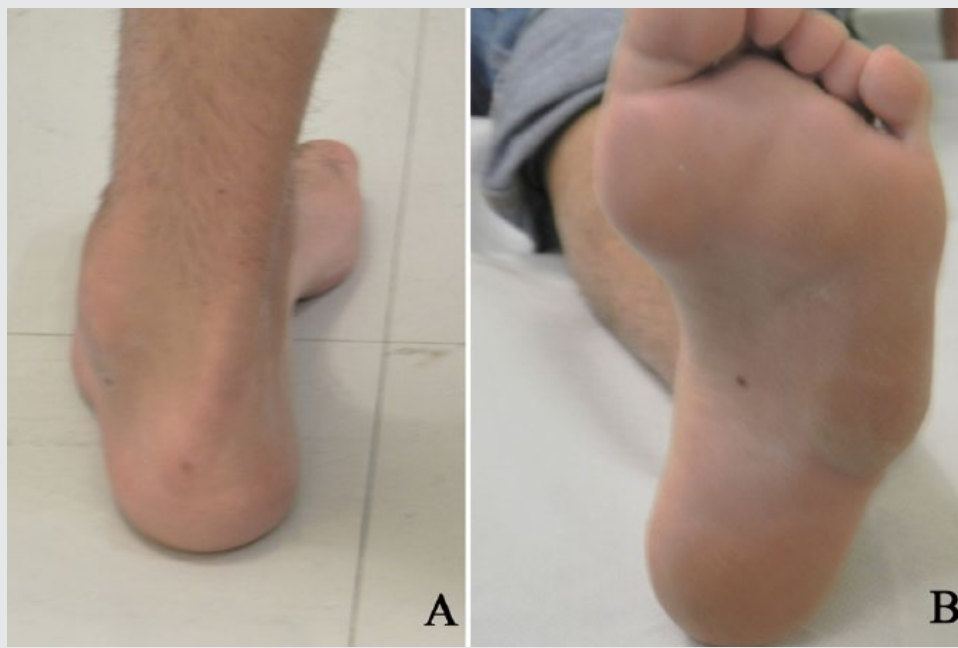

Figure 4.

Case 3: Oblique X-ray showing pseudoarthrosis secondary to stress fracture (A). At 6 months after surgery, oblique $X$-ray showing a complete healing of the stress fracture (B). After a 2-year follow-up, the stress fracture remains consolidated (C).
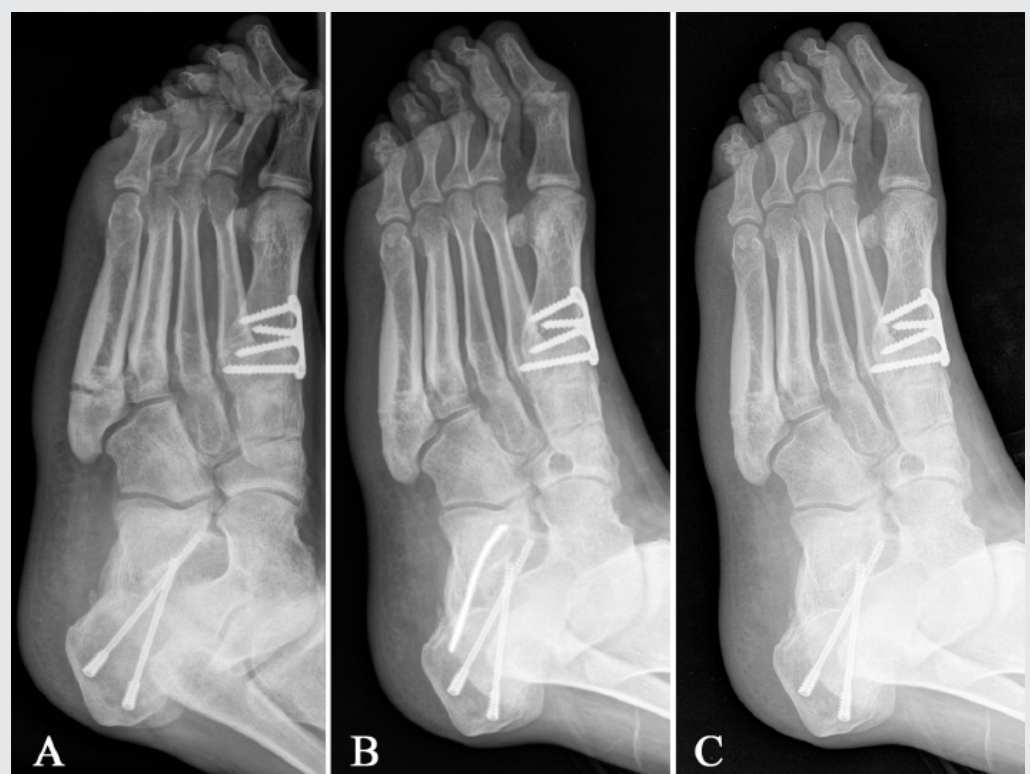

deformity. Gutekunst and Sinacore ${ }^{17}$ detected changes in volumetric bone mineral density, bone geometry, joint malalignment, and biomechanical loads preceding fracture in 2 patients with Charcot neuroarthropathy. They concluded that a plantar load increase on the lateral midfoot following progression of an equine and varus deformity could be causally related to stress fracture of the fifth metatarsal bone.

Treatment of an equinovarus foot deformity secondary to neurological disease should be individualized.

Obtaining a plantigrade and biomechanically functioning foot would be necessary to achieve good muscular balance of the foot. Among the various technical options available, tenodesis, correction osteotomies, arthrodesis, and tendon transfer can be used alone or in combination. In all our cases, a posterior tibialis tendon transfer to the dorsolateral part of the midfoot was associated with arthrodesis and/or corrective osteotomies, allowing a diminishing of overload at any given point. Any of these procedures alone may not be efficient enough to help recover normal-like mechanics. A posterior tibialis tendon transfer would be the key to achieving the best plausible result because it manages the dynamic factor of inversion and plantar flexion that exists in these deformities, simultaneously enabling some dorsiflexion of the foot. ${ }^{18}$

Our patients had stress fractures secondary to equinovarus hindfoot deformity. A surgical correction of the deformity—as we did—should be prioritized over consolidation of the fracture owing to the fact that treating the cause-the deformity - and not the consequence-the fracture-may result in successful fracture healing and a better functioning of the foot. If we attempt to treat the fracture alone without attending to the deformity, refracture or pseudoarthrosis will occur. When we treat the deformity and obtain a plantigrade foot, the high pressure applied on the fifth metatarsal vanishes. Subsequently, the stress that causes the fracture and inhibits the union also disappears.

Some surgeons may consider corrective surgery too aggressive to treat a fracture. Nevertheless, the relationship among a foot cavovarus deformity, a stress fracture, a fracture recurrence, or pseudoarthrosis has long been 


\section{Figure 5.}

Case 3: Physical examination showing a plantigrade alignment on both feet after a 2-year follow-up for the left foot and 1 year for the right foot.

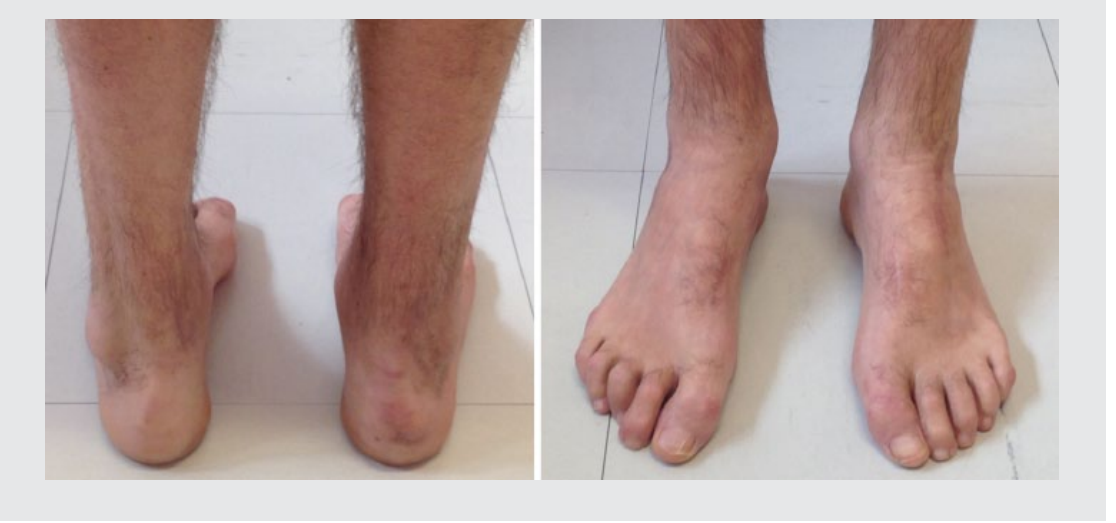

established in the literature. ${ }^{15,19,20}$ In our opinion, stress fracture in the fifth metatarsal secondary to equinovarus deformity should be first treated conservatively with plaster allowing weight bearing, as in our cases. As a result, if there is a refracture or pseudoarthrosis, it can be assumed that a mechanical factor is preventing consolidation, and therefore, changing the foot morphology to obtain a plantigrade foot should be indicated.

Craigen and Clarke ${ }^{21}$ described the case of a 3.5-year-old boy who had a bilateral "Jones" fracture secondary to congenital varus hindfoot. He was treated conservatively for 3 weeks, and union of the fracture was achieved. Two days later this same patient suffered a refracture in the right foot, which was treated conservatively yet again. Then, 17 months later, he presented with a refracture in the left foot, and fracture union was established by means of conservative treatment. McDade et $\mathrm{al}^{22}$ reported 1 metatarsal fracture in a 45-year-old man affected by Parkinson's disease with related levodopa dystonia. Conservative treatment failed. The authors provided no data on the ultimate clinical outcome of the patient. Chee and Walsh ${ }^{20}$ described a girl with an equinovarus deformity secondary to cerebral palsy. Jones's fracture was diagnosed. Initially, she was treated conservatively. Two months later, she refractured over the same site without any trauma. They, then, decided to correct her foot deformity, and the fracture site showed a bony union 5 months later.

Except for the McDade et $\mathrm{al}^{22}$ case report of a spastic-dystonic foot of different etiology and despite the fact that experienced surgeons have classical concepts leading to a solution in these kinds of fractures, we have been able to find 1 sole similar case in the reviewed literature. McDade et $\mathrm{al}^{22}$ did not succeed in healing the fracture and did not consider treating the consolidation failure or improving the biomechanical function of the neurological foot.

This study is limited by the nature of the case reports, which provide the experience of particular cases. However, it may well be a useful tool for readers if they come across a similar pattern, especially taking into account that the recent literature does not offer reliable information regarding a therapeutic orientation.

The clinical relevance of our report is to propose a reflection on the very likely possibility of therapeutic failure when treating stress fractures occurring in patients with neurological hindfoot deformities. We suggest that if the fracture is caused by a secondary deformity, treatment of the deformity should be considered first because it may result in consolidation of the fracture without the need for subsequent surgery on the fracture itself. Conversely, primary treatment of the fracture without accompanying correction of the deformity will lead-as a rule-to therapeutic failure in neurological patients. Future studies are needed to establish the optimal treatment of stress fracture in the fifth metatarsal secondary to neuromuscular disease.

\section{Declaration of Conflicting Interests}

The author(s) declared no potential conflicts of interest with respect to the research, authorship, and/or publication of this article.

\section{Funding}

The author(s) received no financial support for the research, authorship, and/or publication of this article.

\section{Ethical Approval}

Not applicable, because this article does not contain any studies with human or animal subjects.

\section{Informed Consent}

Not applicable, because this article does not contain any studies with human or animal subjects.

\section{Trial Registration}

Not applicable, because this article does

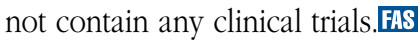

\section{References}

1. Petrisor BA, Ekrol I, Court-Brown C. The epidemiology of metatarsal fractures. Foot Ankle Int. 2006;27:172-174.

2. Kane JM, Sandrowski K, Saffel H, Albanese A, Raikin SM, Pedowitz DI. The epidemiology of fifth metatarsal fracture. Foot Ankle Spec. 2015;8:354-359.

3. Jones R. Fracture of the base of the fifth metatarsal bone by indirect violence. Ann Surg. 1902;35:697-700.2.

4. Zelko RR, Torg JS, Rachun A. Proximal diaphyseal fractures of the fifth 
metatarsal-treatment of the fractures and their complications in athletes. Am J Sports Med. 1979;7:95-101.

5. McKeon KE, Johnson JE, McCormick JJ, Klein SE. The intraosseous and extraosseous vascular supply of the fifth metatarsal: implication for fifth metatarsal osteotomy. Foot Ankle Int. 2013;34:117-123.

6. Carp L. Fracture of the fifth metatarsal bone: with special reference to delayed union. Ann Surg. 1927;86:308-320.

7. Shereff MJ, Yang QM, Kummer FJ, Frey CC, Greenidge N. Vascular anatomy of the fifth metatarsal. Foot Ankle. 1991;11:350-353.

8. Chuckpaiwong B, Queen RM, Easley ME, Nunley JA. Distinguishing Jones and proximal diaphyseal fractures of the fifth metatarsal. Clin Orthop Relat Res. 2008;466:1966-1970.

9. Rosenberg GA, Sferra JJ. Treatment strategies for acute fractures and nonunions of the proximal fifth metatarsal. J Am Acad Orthop Surg. 2000;8:332-338.

10. Ding BC, Weatherall JM, Mroczek KJ, Sheskier SC. Fracture of the proximal fifth metatarsal: keeping up with the Joneses. Bull NYU Hosp Jt Diss. 2012;70:49-55.
11. Kavanaugh JH, Brower TD, Mann RV. The Jones fracture revisited. J Bone Joint Surg Am. 1978;60:776-782.

12. DeLee JC, Evans JP, Julian J. Stress fracture of the fifth metatarsal. Am J Sports Med. 1983;11:349-353.

13. Yates J, Feeley I, Sasikumar S, Rattan G, Hannigan A, Sheehan E. Jones fracture of the fifth metatarsal: is operative intervention justified? A systematic review of the literature and meta-analysis of results. Foot (Edinb). 2015;25:251-257.

14. Wright RW, Fischer DA, Shively RA, Heidt RS Jr, Nuber GW. Refracture of proximal fifth metatarsal (Jones) fracture after intramedullary screw fixation in athletes. Am J Sports Med. 2000;28:732736.

15. Raikin SM, Slenker N, Ratigan B. The association of a varus hindfoot and fracture of the fifth metatarsal metaphysealdiaphyseal junction: the Jones fracture. $A m$ J Sports Med. 2008;36:1367-1372.

16. Lee KT, Park YU, Jegal H, Kim KC, Young KW, Kim JS. Factors associated with recurrent fifth metatarsal stress fracture. Foot Ankle Int. 2013;34:1645-1653.
17. Gutekunst DJ, Sinacore DR. Pedal bone density, strength, orientation, and plantar loads preceding incipient metatarsal fracture after Charcot neuroarthropathy: 2 case reports. J Orthop Sports Phys Ther. 2013;43:744-751.

18. Dreher T, Wolf SI, Heitzmann D, Fremd C, Klotz MC, Wenz W. Tibialis posterior tendon transfer corrects the foot drop component of cavovarus foot deformity in Charcot-Marie-Tooth disease. J Bone Joint Surg Am. 2014;96:456-462.

19. Boutefnouchet $T$, Budair B, Backshayesh P, Ali SA. Metatarsal fractures: a review and current concepts. Trauma. 2014;16:147163.

20. Chee Y, Walsh HPJ. The Robert Jones fracture - a lesson learned. Foot Ankle Surg. 2005;11:59-61.

21. Craigen MA, Clarke NM. Bilateral "Jones" fractures of the fifth metatarsal following relapse of talipes equinovarus. Injury. 1996;27:599-601.

22. McDade E, Weiner WJ, Shulman LM. Metatarsal fracture as a consequence of foot dystonia in Parkinson's disease. Parkinsonism Relat Disord. 2008;14:353-355. 